The Astrophysical Journal, 631:L147-L150, 2005 October 1

(C) 2005. The American Astronomical Society. All rights reserved. Printed in U.S.A.

\title{
R CrB CANDIDATES IN THE SMALL MAGELLANIC CLOUD: OBSERVATIONS OF COLD, FEATURELESS DUST WITH THE SPITZER INFRARED SPECTROGRAPH
}

\author{
Kathleen E. Kraemer, ${ }^{1}$ G. C. Sloan, ${ }^{2}$ P. R. Wood, ${ }^{3}$ Stephan D. Price, ${ }^{1}$ and Michael P. Egan ${ }^{1}$ \\ Received 2005 July 29; accepted 2005 August 25; published 2005 September 13
}

\begin{abstract}
We observed 36 evolved stars in the Small Magellanic Cloud (SMC) using the low-resolution mode of the Infrared Spectrograph (IRS) on the Spitzer Space Telescope. Two of these stars, MSX SMC 014 and 155, have nearly featureless spectral energy distributions over the IRS wavelength range $(5.2-35 \mu \mathrm{m})$ and $F_{\nu}$ peaking at $\sim 8-9 \mu \mathrm{m}$. The data can be fit by sets of amorphous carbon shells or by single $600-700 \mathrm{~K}$ blackbodies. The most similar spectra found in extant spectral databases are of $\mathrm{R} \mathrm{CrB}$, although the spectral structure seen in $\mathrm{R} \mathrm{CrB}$ and similar stars is much weaker or absent in the SMC sources. Both SMC stars show variability in the nearinfrared. Ground-based visual spectra confirm that MSX SMC 155 is carbon-rich, as expected for R CrB (RCB) stars, and coincides with an object previously identified as an RCB candidate. The temperature of the underlying star is lower for MSX SMC 155 than for typical RCB stars. The strength of the $\mathrm{C}_{2}$ Swan bands and the low temperature suggest that it may be a rare DY Per-type star, only the fifth such identified. MSX SMC 014 represents a new RCB candidate in the SMC, bringing the number of RCB candidates in the SMC to six. It is the first RCB candidate discovered with Spitzer and the first identified by its infrared spectral characteristics rather than its visual variability.
\end{abstract}

Subject headings: circumstellar matter — Magellanic Clouds — stars: variables: other

\section{INTRODUCTION}

$\mathrm{R}$ Coronae Borealis (RCB) stars are a rare type of hydrogendeficient, carbon-rich, evolved star that undergo strong, irregular brightness variations in the optical (see Clayton 1996 for a detailed review). Only about 50 or so RCB stars or RCB candidates have been identified in the Milky Way, less than a score in the Large Magellanic Cloud (LMC; Alcock et al. 1996b, 2001), and five in the Small Magellanic Cloud (SMC; Morgan et al. 2003; Tisserand et al. 2004). Distances to Galactic RCB stars are problematic, and thus distant-dependent properties of these poorly understood objects, such as absolute luminosity, are also problematic. Distances to the LMC and SMC, in contrast, are well constrained. Identification and observation of RCB candidates in the LMC and SMC are therefore essential for characterizing and understanding this phase of stellar evolution.

\section{OBSERVATIONS}

We observed MSX SMC 014 and 155 (following the nomenclature of Egan et al. 2001), ${ }^{4}$ two evolved stars in the SMC, with the Infrared Spectrograph (IRS; Houck et al. 2004) on the Spitzer Space Telescope (Werner et al. 2004) on 2004 October 25. The Two Micron All Sky Survey (2MASS) positions $(\mathrm{J} 2000)$ are R.A. $=00^{\mathrm{h}} 46^{\mathrm{m}} 16^{\mathrm{s}} 33$, decl. $=-74^{\circ} 11^{\prime} 13^{\prime \prime} \cdot 6$ and R.A. $=00^{\mathrm{h}} 57^{\mathrm{m}} 18 \mathrm{~s} 15$, decl. $=-72^{\circ} 42^{\prime} 35^{\prime \prime} \cdot 2$ for MSX SMC 014 and 155, respectively. The observations used the ShortLow (SL) and Long-Low (LL) modules, which have a wavelength range of 5.2-35 $\mu \mathrm{m}$ and a spectral resolution of $\sim 100$. We extracted the spectra from the flat-fielded images produced

\footnotetext{
${ }^{1}$ Air Force Research Laboratory, Space Vehicles Directorate, 29 Randolph Road, Hanscom AFB, MA 01731; kathleen.kraemer@hanscom.af.mil, steve .price@hanscom.af.mil, michael.egan@osd.mil.

${ }^{2}$ Department of Astronomy, Cornell University, 108 Space Sciences Building, Ithaca, NY 14853; sloan@astro.cornell.edu.

${ }^{3}$ Research School of Astronomy and Astrophysics, Mount Stromlo Observatory, Weston Creek, ACT 2611, Australia; wood@mso.anu.edu.au.

${ }^{4}$ In the Midcourse Space Experiment (MSX) Point Source Catalog, ver. 2.3 (Egan et al. 2003), MSX SMC $014=$ G303.4131-42.9358, and MSX SMC $155=$ G302.3214-44.4109.
}

by the Spitzer Science Center pipeline S11.0 using the software available with the Spitzer IRS Custom Extractor. The spectra from each order and nod were extracted separately and fluxcalibrated with HR 6348 for SL and HR 6348, HD 166780, and HD 173511 for LL (see G. C. Sloan et al. 2005, in preparation). Errors were estimated by comparing the nods, and multiplicative corrections were used to remove discontinuities between orders caused by random pointing offsets. Figure 1 shows the resulting, featureless spectra, which look remarkably like blackbody curves.

Optical spectra of MSX SMC 155 and two comparison stars were taken on 2004 November 22 ( 28 days after the IRS data) using the Double Beam Spectrograph on the $2.3 \mathrm{~m}$ telescope of the Australian National University at Siding Spring Observatory (SSO). The spectral range was $0.45-1.05 \mu \mathrm{m}$, and the resolution was $10 \AA$. The data were reduced with standard IRAF procedures using HR 718 as a flux standard and the weak-lined giant HD 26169 to remove telluric features. Due to some cloudiness during the observations, the relative flux calibration is correct, but the absolute flux is uncertain. In addition to the optical spectra, $J H K L$ photometry was taken of both stars on 2004 November 25 using the cryogenic array spectrometer/imager (CASPIR) on the $2.3 \mathrm{~m}$ telescope. They have since been monitored periodically at the $K$ band (see Tables 1 and 2).

\section{3. $\mathrm{R}$ CrB STAR CANDIDATES}

Evolved stars can generally be divided into oxygen-rich and carbon-rich groups, with oxygen-rich stars usually showing mid-IR silicate or alumina dust features and carbon stars showing features from $\mathrm{SiC}$ dust and polycyclic aromatic hydrocarbons (PAHs), and bands from $\mathrm{C}_{2} \mathrm{H}_{2}$ and other carbon-bearing molecules. In contrast, the mid-IR spectra of MSX SMC 014 and 155 are virtually featureless, offering few clues as to the chemistry of their dust, let alone their photospheres.

We examined spectra from the Short Wavelength Spectrometer (SWS) and the Photo-Polarimeter on the Infrared Space Observatory, looking for similarly bland spectral energy distributions (SEDs) where $F_{\nu}$ peaks at $\sim 7-9 \mu \mathrm{m}$, which led us to 


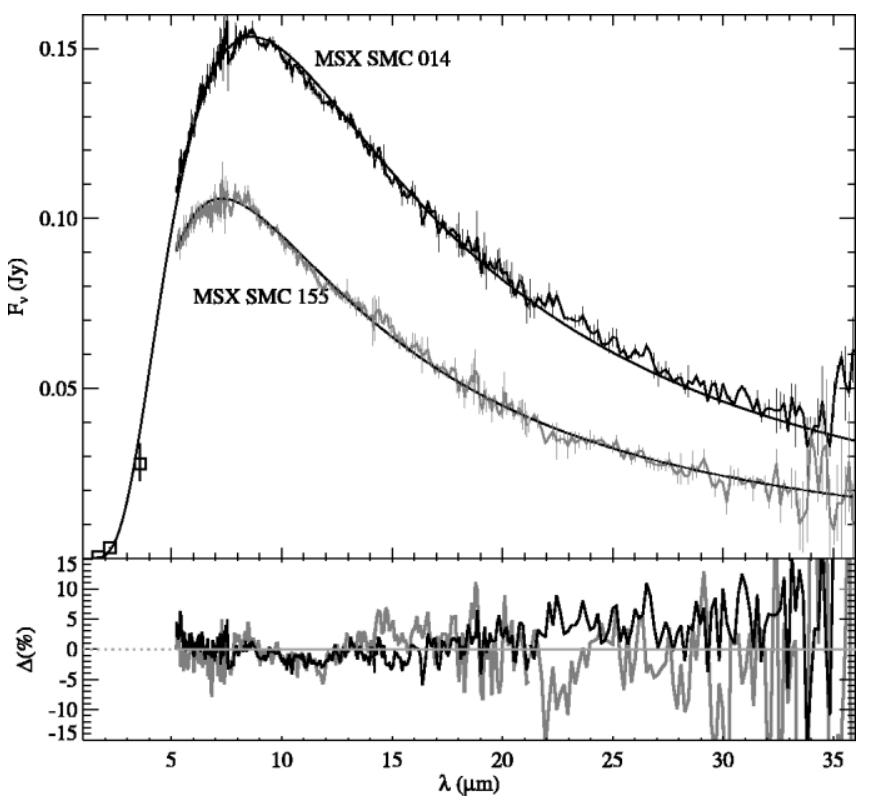

FIG. 1.-Top: IRS full-range spectra for MSX SMC 014 (black) and 155 (gray), including uncertainties. The smooth curves show the blackbody functions fit to the IRS data: $590 \mathrm{~K}$ for MSX SMC 014 and $700 \mathrm{~K}$ for MSX SMC 155 (§ 4). Bottom: Residuals (\%) between the data and the blackbody fits. The gray line shows the residuals for MSX SMC 155, and the black line for MSX SMC 014. The squares in the top panel are the near-IR SSO data for MSX SMC 014 (see text).

spectra in the 3.W class of Kraemer et al. (2002). Closer inspection revealed that the objects in 3 .W without silicate absorption are mostly RCB stars (those with silicate absorption are usually Wolf-Rayet stars). Figure 2 compares the SWS spectrum of R CrB itself and the IRS spectra of MSX SMC 014 and 155. R CrB shows broad emission features around 6 and $14 \mu \mathrm{m}$ that were identified by Lambert et al. (2001) as amorphous carbon features. The spectra of the SMC stars agree quite well with the $\mathrm{R} \mathrm{CrB}$ data, although they have at best very weak features at 6 and $14 \mu \mathrm{m}$. The lack of hydrogen in RCB stars naturally leads to the formation of amorphous carbon dust (e.g., Hecht et al. 1984), which is nearly featureless in the IR, instead of hydrogen-rich particles such as PAHs, which have strong IR features that are not present in the SMC data.

Most (if not all) RCB candidates have previously been identified by their strong and irregular variability in the visible. Tisserand et al. (2004) noted such variability in MSX SMC 155 and identified it as a candidate RCB star based on the 56 mag variation in EROS 2 data (Expérience de Recherche d'Objets Sombres). ${ }^{5}$ We confirmed the carbon-rich nature of the central star of MSX SMC 155 with visual spectroscopic observations, as Figure 3 shows. Our multiepoch $K$-band data, shown in Figure 4 along with the 2MASS $K_{s}$ datum from 1998, indicate that MSX SMC 155 is variable in the near-IR as well

${ }^{5}$ EROS name: J005718-724235 (sm0067m2813b); MACHO identifier: 207.16426.1662. as the visible. The timescale and magnitude of the $K$-band variation in MSX SMC 155 (1 mag decline in 240 days) are fairly typical of RCB stars (Feast et al. 1997) as are the nearIR colors (Feast 1997). Thus, our visual and IR observations strongly support the candidacy of MSX SMC 155 as an RCB star.

MSX SMC 014 does not appear in any of the databases produced by EROS or other dark matter searches that target the Magellanic Clouds (e.g., Alcock et al. 1996a; Szymański 2005), being outside their observed fields. Due to its dimness, we were unable to confirm its carbon-rich nature with groundbased spectroscopy. We did detect $K$-band variability (Fig. 4), as with MSX SMC 155. The recent variation is not as great as MSX SMC 155 showed (0.96 mag vs. $0.28 \mathrm{mag}$ ), but there has been an $\sim 0.7$ mag increase in brightness since the 2MASS observation in 1998. It appears that both MSX SMC 155 and 014 were near maximum $K$ brightness when observed by Spitzer, with MSX SMC 155 starting a fading event about 3060 days after the Spitzer observation (Fig. 4). MSX SMC 014 was redder than the stars studied by Feast et al. (1997), but it is similar to the LMC RCB star HV 2379 during its faded period (Bessell \& Wood 1983). Given the similarity of the midIR spectrum of MSX SMC 014 to those of MSX SMC 155 and $\mathrm{R} \mathrm{CrB}$, as well as the near-IR variability, we feel confident in identifying it as the sixth RCB candidate in the SMC. It is also the first identified by its IR spectrum (as opposed to visual variability) and the first identified by Spitzer.

\section{DUST AND STELLAR PROPERTIES}

The near featurelessness of the spectra precludes a significant contribution from grains with strong spectral structure, such as graphite or silicates, which dominate the mid-IR dust spectra of ordinary evolved stars. Thus, we initially fit single-temperature Planck functions to the spectra, which are shown in Figure 1. MSX SMC 014 can be fit with curves in the temperature range $580-610 \mathrm{~K}$, with the best fit at $590 \pm 5 \mathrm{~K}$. MSX SMC 155 is best fit with a $700 \pm 5 \mathrm{~K}$ blackbody but could be as cool as $690 \mathrm{~K}$. The residuals left after subtraction of the indicated blackbody from the IRS data are less than $10 \%$ for most of the IRS wavelength range (Fig. 1). Dust temperatures for Galactic RCB stars typically lie in the range 600-900 K (Kilkenny \& Whittet 1984; Walker 1986), so the single-component dust temperatures of the SMC objects are comparable to the Galactic population.

Amorphous carbon, relatively featureless in the mid-IR as noted above, is a likely carrier, so we obtained optical constants from Suh (2000) and Zubko et al. (1996). ${ }^{6}$ Although mostly featureless, the amorphous carbon SEDs are significantly narrower than the observed spectra, if the grains are assumed to be at a single temperature (i.e., at a single distance from the central star). A simple model, though, with a set of SEDs from amorphous carbon "shells" at varying distances from the central

\footnotetext{
${ }^{6}$ The optical constants are available from their respective Web sites http://ast.chungbuk.ac.kr/ kwsuh/d-opt.htm and http://idlastro.gsfc.nasa.gov/ $\sim$ zubko/nk.html.
}

TABLE 1

NeAR-IR PHOTOMETRY

\begin{tabular}{ccccc}
\hline \hline & \multicolumn{5}{c}{ 2004 November 25 } \\
\cline { 2 - 5 } StAR & $J$ & $H$ & $K$ & $L$ \\
\hline MSX SMC 014 $\ldots \ldots$ & $\ldots$ & $15.683 \pm 0.093$ & $13.212 \pm 0.021$ & $9.939 \pm 0.211$ \\
MSX SMC $155 \ldots \ldots$ & $13.740 \pm 0.064$ & $12.548 \pm 0.010$ & $11.370 \pm 0.016$ & $9.144 \pm 0.090$
\end{tabular}


TABLE 2

Multiepoch $K$-Band Photometry

\begin{tabular}{ccccc}
\hline \hline & \multicolumn{4}{c}{$K$ BAND } \\
\cline { 2 - 5 } STAR & 2004 Nov 25 & 2005 Jan 26 & 2005 Mar 14 & 2005 Jul 24 \\
\hline MSX SMC 014 ...... & $13.212 \pm 0.021$ & $13.362 \pm 0.021$ & $13.284 \pm 0.052$ & $13.493 \pm 0.024$ \\
MSX SMC 155 ...... & $11.370 \pm 0.016$ & $11.522 \pm 0.014$ & $12.003 \pm 0.024$ & $12.327 \pm 0.012$ \\
\hline
\end{tabular}

star can fit the IRS data as well as the single-temperature blackbodies. (Note that the shells are simple representations of the irregular dust condensations that likely cause the visible obscuration and are not intended as actual physical entities.) The temperature range for the dust shells is about $500-1100 \mathrm{~K}$ (Fig. 5). However, the IRS data do little to constrain the stellar temperature since, for instance, a hotter star can be compensated for by placing the first shell at a greater distance from the star.

The near-IR data, though, do provide constraints on the stellar temperature and, to a lesser extent, the position of the inner shell, its visual opacity, and the number of shells. (Complete modeling to explore the full parameter space is deferred to a future paper.) For MSX SMC 014, the $K$ - and $L$-band points lie on the $590 \mathrm{~K}$ blackbody (Fig. 1) or multishell amorphous carbon curves. The $H$-band point may be slightly above the curves, which may indicate tentative detection of a photospheric contribution (the $J$ band is an upper limit in both the SSO and 2MASS data) but by itself is insufficient to constrain the models. The near-IR data for MSX SMC 155, though, lie well above the extrapolated mid-IR curves, so we do detect a photospheric contribution. For the amorphous carbon shells, a small contribution from stellar temperatures of $\sim 2000-2500 \mathrm{~K}$ is supported by the near-IR data; higher temperatures produce too much $J$-band flux but underestimate the $K_{s}$-band flux. For the single-temperature dust model, slightly higher stellar tem-

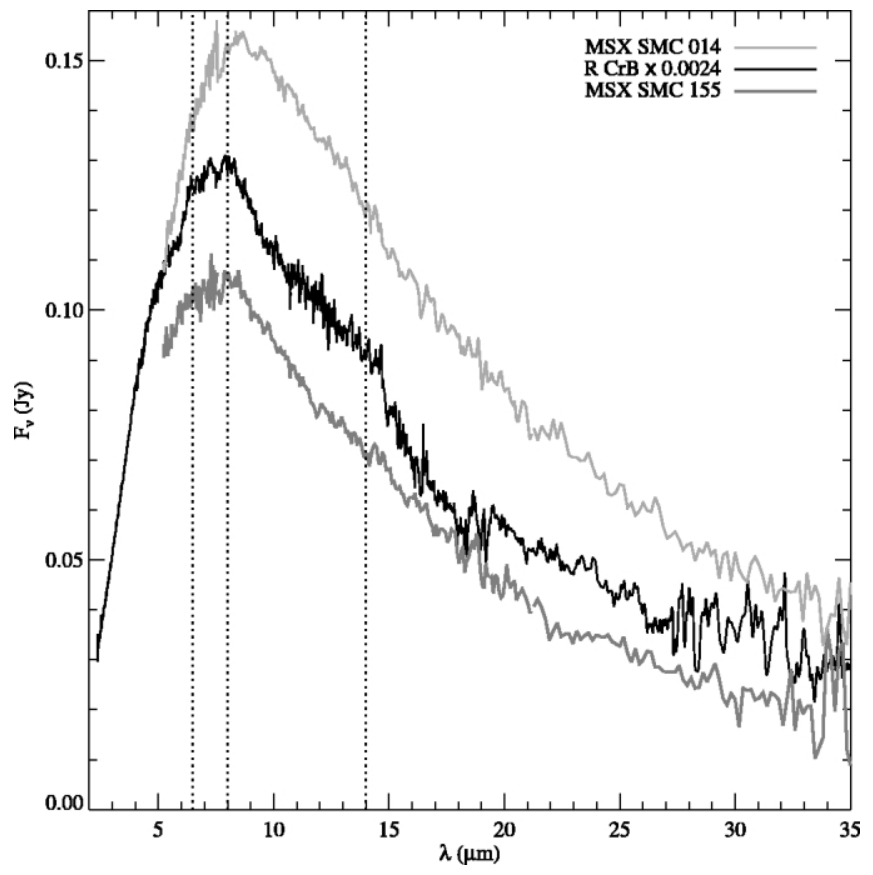

FIG. 2.-The top trace (light gray) shows MSX SMC 014; the middle trace (black) shows R CrB (Sloan et al. 2003), scaled by 0.0024; and the bottom trace (dark gray) shows MSX SMC 155. The dotted lines indicate the regions of possible spectral features: amorphous carbon at 6 and $14 \mu \mathrm{m}$ in $\mathrm{R} \mathrm{CrB}$ (Lambert et al. 2001) and a possible, unidentified feature near $8 \mu \mathrm{m}$ in all three. peratures fit, although this is a less physically plausible model. Figure 5 shows typical results for the fitted models. The ranges in stellar temperature estimated for MSX SMC 155 are colder than even the unusually cool RCB star DY Per $(\sim 3500 \mathrm{~K}$; Keenan \& Barnbaum 1997).

Comparison of the optical spectrum of MSX SMC 155, particularly the $5635 \AA$ band of $\mathrm{C}_{2}$, with the catalog of $\mathrm{C}$ star spectra of Barnbaum et al. (1996) and the $T_{\text {eff }}$ values given for the catalog stars by Bergeat et al. (2001) suggests that $T_{\text {eff }}$ for MSX SMC 155 is most likely $\sim 3000-4000 \mathrm{~K}$, although cooler values down to $2500 \mathrm{~K}$ cannot be ruled out. The strong $\Delta v=3$ vibrational band heads of $\mathrm{C}_{2}$ in MSX SMC 155 (6526$6854 \AA$ ) suggest that $C_{2}$ has been excited to unusually high vibrational levels. These band heads are weak or absent in typical C stars (e.g., MSX SMC 134 in Fig. 3; Barnbaum et al. 1996; Alcock et al. 2001) but were also strong in HV 2379 (Bessell \& Wood 1983). Finally, our visual spectrum is quite similar to those of the four LMC RCB stars of Alcock et al. (2001) that have 4000-7000 ̊ spectra resembling DY Per. We suggest that MSX SMC 155, too, may be a cool, DY Per-type RCB star.

The distance to the SMC is known (60 kpc or a distance modulus of 18.9), so we can calculate the luminosities. We integrated under the single-temperature (590 K) blackbody fit for MSX SMC 014 and a model of amorphous carbon shells plus a $2500 \mathrm{~K}$ blackbody (the dotted line in Fig. 5) for MSX SMC 155. These gave luminosities of $L \approx 9700 L_{\odot}$ and $L \approx$ $11000 L_{\odot}$ of which roughly $70 \%$ and $40 \%$ emerge within the IRS bandpass for MSX SMC 014 and 155, respectively. Given

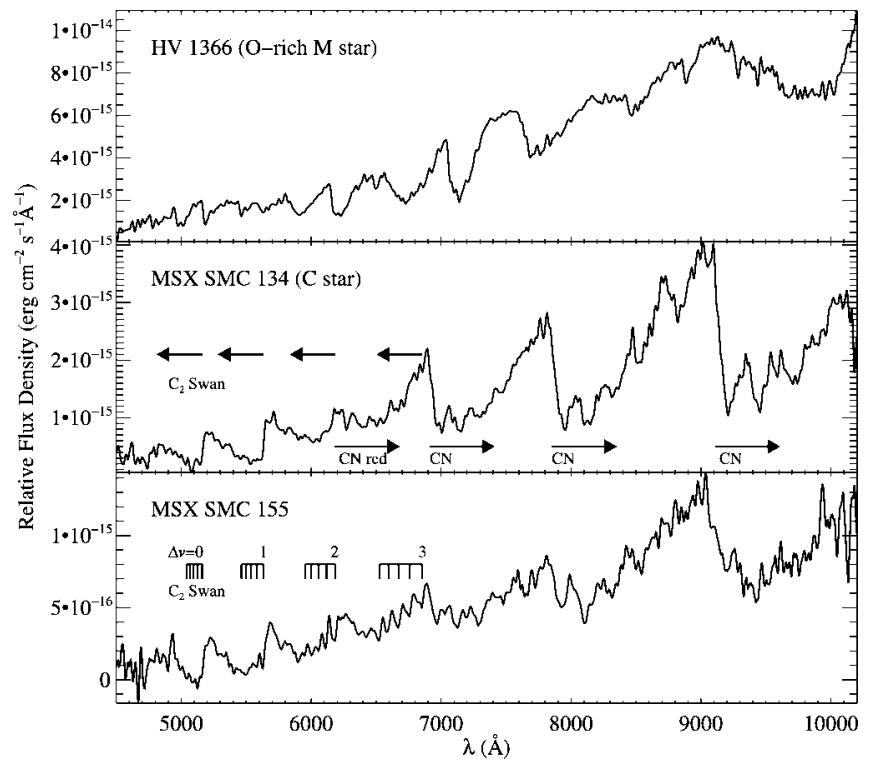

FIG. 3.-The visual spectrum of MSX SMC 155 (bottom) is a much better match to the carbon star MSX SMC 134 (middle) than the O-rich M star HV 1366 (top). The $\mathrm{C}_{2}$ Swan bands and $\mathrm{CN}$ red bands are indicated on the MSX SMC 134 plot. Individual vibrational band heads for the Swan bands are shown in the MSX SMC 155 panel, which has particularly strong $\Delta v=3$ bands. 


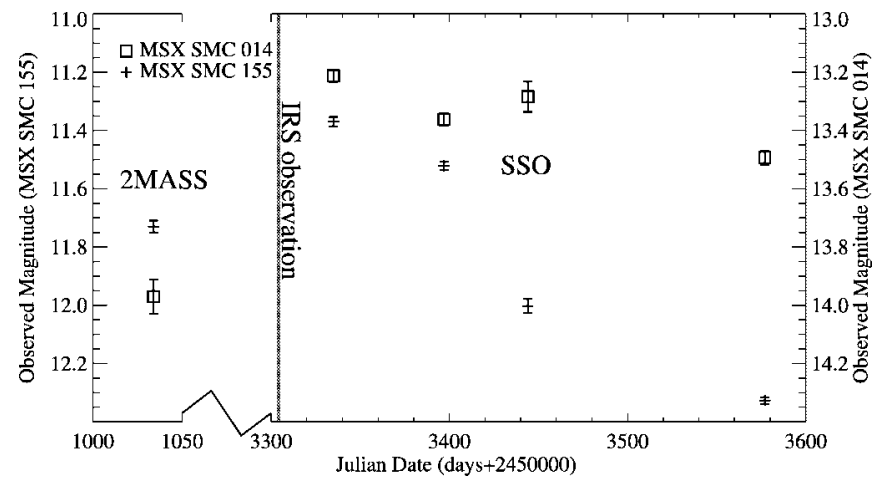

FIG. 4.- $K$-band observations of MSX SMC 155 (plus signs) and MSX SMC 014 (squares). The first set of points are the 2MASS $K_{s}$ data; the rest are the more recent observations from the SSO (note the break in $x$-axis scale). The $y$-scale on the left side corresponds to MSX SMC 155, and that on the right side is for MSX SMC 014. Error bars are $1 \sigma$ measurement uncertainties. The thick gray line indicates the date of the IRS observations (JD 2,453,304).

these luminosities and the cool photospheric temperatures suggested for the central stars, it appears that these objects may still be on the asymptotic giant branch (AGB) but are undergoing the same type of episodic carbon shell ejections that are seen in warmer RCB stars.

\section{SUMMARY}

We observed two objects in the SMC with largely featureless spectra in the mid-IR. These spectra are most similar to the SWS data for R CrB and other Galactic RCB stars. MSX SMC 155 has been identified based on its optical variability as one of five RCB candidates in the SMC. The second star, MSX SMC 014, has no readily available visual photometry, but our $K$-band observations, along with the 2MASS data, indicate that it is a near-IR variable. Based on the similarity of its IRS spectrum to that of MSX SMC 155, we identify MSX SMC 014 as the first RCB candidate found by its IR spectral properties rather than by optical variability, and also as the first RCB candidate discovered by Spitzer. The spectra can be fit by either a single-temperature blackbody or a set of amorphous carbon shells produced by different episodes of dust formation. Calculated luminosities and estimated stellar temperatures im-

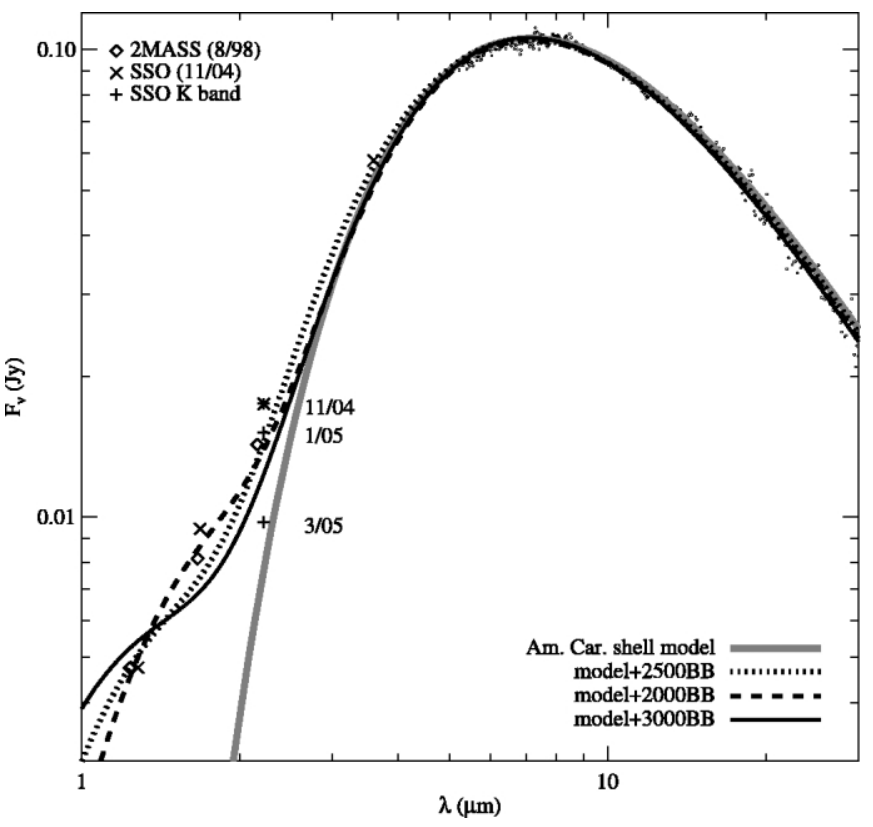

FIG. 5.-IRS spectrum (small circles) for MSX SMC 155 plus the 2MASS data (diamonds), the SSO (crosses) data, and the multiepoch $K$-band data from the SSO (plus signs with dates). The thick gray line shows the model for a $2500 \mathrm{~K}$ central star with 12 shells of amorphous carbon starting at $R=$ $6.1 R_{*}$. The dotted line adds a small contribution from a $2500 \mathrm{~K}$ blackbody. The dashed line shows a similar shell+blackbody model, but for a $2000 \mathrm{~K}$ star, and the black line is for a $3000 \mathrm{~K}$ star.

ply that the central star for MSX SMC 155 may be a carbon star still on the AGB. We suggest that it is the first DY Per star, a rare type of very cool RCB star, identified in the SMC.

We wish to thank Bill Forrest for useful discussions on amorphous carbon shells and the referee, Geoffrey Clayton, whose helpful suggestions improved this Letter. This work is based in part on observations made with the Spitzer Space Telescope, which is operated by JPL/Caltech under NASA contract 1407. Support for this work was provided in part by NASA; P. R. W. received funding for this work from the Australian Research Council. This research has made use of NASA's Astrophysics Data System, 2MASS, and SIMBAD.

\section{REFERENCES}

Alcock, C., et al. 1996a, ApJ, 461, 84

1996b, ApJ, 470, 583

2001, ApJ, 554, 298

Barnbaum, C., Stone, R. P. S., \& Keenan, P. C. 1996, ApJS, 105, 419

Bergeat, J., Knapik, A., \& Rutily, B. 2001, A\&A, 369, 178

Bessell, M. S., \& Wood, P. R. 1983, MNRAS, 202, 31P

Clayton, G. C. 1996, PASP, 108, 225

Egan, M. P., van Dyk, S. D., \& Price, S. D. 2001, AJ, 122, 1844

Egan, M. P., et al. 2003, The Midcourse Space Experiment Point Source Catalog, Ver. 2.3, Explanatory Guide, Air Force Res. Lab. Tech. Rep. AFRLVS-TR-2003-1589 (Hancom AFB: AFRL)

Feast, M. W. 1997, MNRAS, 285, 339

Feast, M. W., Carter, B. S., Roberts, G., Marang, F., \& Catchpole, R. M. 1997, MNRAS, 285, 317

Hecht, J. H., Holm, A. V., Donn, B., \& Wu, C.-C. 1984, ApJ, 280, 228

Houck, J., et al. 2004, ApJS, 154, 18

Keenan, P. C., \& Barnbaum, C. 1997, PASP, 109, 969
Kilkenny, D., \& Whittet, D. C. B. 1984, MNRAS, 208, 25

Kraemer, K. E., Sloan, G. C., Price, S. D., \& Walker, H. J. 2002, ApJS, 140, 389

Lambert, D. L., Rao, N. K., Pandey, G., \& Ivans, I. I. 2001, ApJ, 555, 925

Morgan, D. H., Hatzidimitriou, D., Cannon, R. D., \& Croke, B. F. W. 2003, MNRAS, 344, 325

Sloan, G. C., Kraemer, K. E., Price, S. D., \& Shipman, R. F. 2003, ApJS, 147,379

Suh, K.-W. 2000, MNRAS, 315, 740

Szymański, M. 2005, Acta Astron., 55, 43

Tisserand, P., et al. 2004, A\&A, 424, 245

Walker, H. J. 1986, in IAU Colloq. 87, Hydrogen Deficient Stars and Related Objects, ed. K. Hunger, D. Schönberner, \& N. K. Rao (Dordrecht: Reidel), 407

Werner, M., et al. 2004, ApJS, 154, 1

Zubko, V. G., Mennella, V., Colangeli, L., \& Bussoletti, E. 1996, MNRAS, 282,1321 